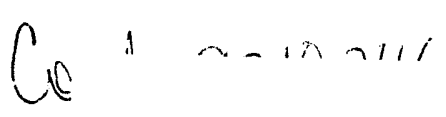

ANL/ASD/CP-77498

DE93 004182

\title{
FLUORESCENT SCREENS AND IMAGE PROCESSING FOR THE APS LINAC TEST STAND*
}

\author{
W. Berg and K. Ko \\ Argonne National Laboratory, Advanced Photon Source \\ 9700 South Cass Avenue \\ Argonne, IL 60439

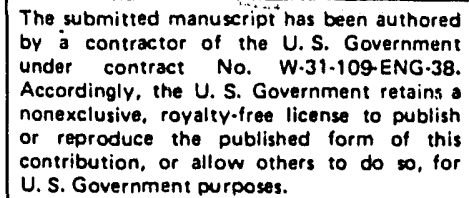

A fluorescent screen was used to monitor relative beam position and spot size of a $56 \cdot \mathrm{MeV}$ electron beam in the linac test stand. A chromium doped alumina ceramic screen inserted into the beam was monitored by a video camera. The resulting image was captured using a frame grabber and stored into memory. Reconstruction and analysis of the stored image was performed using PV.WAVE ${ }^{1}$. This paper will discuss the hardware and software implementation of the fluorescent screen and imaging system. Proposed improvements for the APS linac fluorescent screens and image processing will also be discussed.

\section{INTRODUCTION}

The APS linac test stand produced a $56 \cdot \mathrm{MeV}$ electron beam at a repetition rate of $3 \mathrm{~Hz}$ with a minimum spot size of $6 \mathrm{~mm}$. Knowledge of the beam size and relative position was important for startup beam optimization and diagnostic procedures. These parameters were determined by an analysis of the beam's image formed on a fluorescent screen.

Image processing starts with the beam image being formed on a fluorescent screen made of chromium doped alumina ceramic. A metallic grid pattern evaporated onto the screen removes static charge built up on the surface, and serves as a reference for calibration. This screen is continuously monitored by a frame grabber system which is comprised of commercially available hardware. When a trigger is received, the frame grabber is switched to the acquisition mode to capture the beam image.

A program written in PV.WAVE Command Language is used to perform analysis of the stored image data. This program reconstructs the data and presents the information on a terminal. From the analysis, relative beam position and spot size are determined. Pseudo colors are assigned to the black and white image to help visualize the beam size and shape. A typical analysis result is shown in Figs. 1a through $\mathrm{f}$.

This paper presents a detailed discussion of the hardware and software implementations of the image processing procedure.

\section{FLUORESCENT SCREEN}

The prototype fluorescent screen currently being used is made of a chromium doped alumina ceramic commonly known as Chromox 2 . The physical geometry of the screen is a disk 2-1/2" in diameter and .020 " thick. A metallic grid pattern yielding 1.mm-square increments has been evaporated onto the face of the screen. The grid pattern is used for calibration of the imaging system and to help eliminate surface charge effects stemming from a buildup of charge.

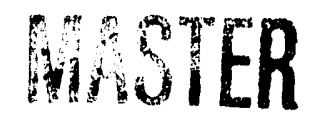


A modified 5-way vacuum cross serves as the housing for the fluorescent screen. There are two quartz windows attached to the vacuum cross. One is used for imaging and the other allows for background illumination during calibration. A pneumatic actuator is used to insert and retract the screen from the beamline. The screen lies in a plane such that its $X$ axis is rotated $45^{\circ}$ with respect to the center of the beamline. Similarly the screen is at an angle of $45^{\circ}$ relative to the view port. The fluorescent screen assembly is pictured in Fig. 2.

\section{FRAME GRABBER}

The frame grabber used in the linac test stand is a VME based system. It includes a black and white video camera and a MaxVideo 10 system with DigiMax ${ }^{3}$ frame grabber and RoiStore ${ }^{4}$ video memory. The camera has an interlaced RS170 analog composite video output which generates 30 frames of video information per second. In normal operation, the video signal is continuously digitized by the frame grabber. When triggered, digitized data from the frame grabber is written to the video memory of RoiStore. Each digitized pixel has an eight-bit resolution which corresponds to 256 different intensity levels. The image data stored is saved in a file for analysis.

Each frame of video information consists of $512 \times 484$ pixels which represent the vertical and horizontal resolutions, respectively. A frame is divided into even and odd fields. The odd field contains all of the odd numbered scan lines while the even field contains the even numbered scan lines. However, the se two fields are not produced at the same time. They alternate with a scan time of $1 / 60$ of a second each and are recombined to form one frame. Because of this time lag, only one of the fields is used during analysis. Due to the hardware implementation chosen the even field was used in this case.

Since there is no external trigger on the camera or the frame grabber, the data acquisition is triggered by a software flag synchronized with the electron gun trigger. When the electron gun is pulsing, the operator can put the frame grabber into acquisition mode. At this point the frame grabber waits for the software flag before starting the frame grabbing process. However, the beam image will not be captured instantaneously because the camera is operating independently. The frame hardware grabber has to wait until the transmission of the current frame is completed. This may introduce a delay of $1 / 30$ of a second in the worst case. There is also another $1 / 30$ of a second delay due to the hardware configuration, therefore a $1 / 30$ to $1 / 15$ of a second timing uncertainty in the captured image with respect to the firing signal may result.

\section{PV-WAVE ANALYSIS}

Data analysis is done with a program written in PV-WAVE Command Language. PV-WAVE is a high-level language software package with functions and procedure calls tc perform visual data analysis. A typical result of the analysis is shown in Figs. 1a through $f$. The raw image, background, reconstructed image with background subtracted, 3-D projection of the intensity of the reconstructed image, and $\mathrm{X}$ and $\mathrm{Y}$ profiles of the intensity at the center of the beam are shown. In order to help visualize 
the shape of the beam, pseudo colors are assigned to different intensity levels. There are four basic functions of the program.

1. Perform background subtraction.

2. Reconstruct the distorted image.

3. Display $\mathrm{X}$ and $\mathrm{Y}$ profiles at the center of the beam.

4. Compensate for grid pattern interference.

Although the beam image is taken with a dark background, it is desirable to perform a background subtraction on the image before any analysis is done. Background subtraction finds the pixel by pixel difference of the raw image and the background image. The advantage of this subtraction is to bring out the beam image from the background by eliminating background noise as can be seen in Fig. 1c.

Since the camera is placed at an angle of $45^{\circ}$ with respect to the screen, the captured beam image has been distorted due to the linear perspective of the projection. The rectangular grid on the screen in Fig. $3 a$ is a typical example. In order to measure the beam's relative position and spot size, the distorted area is reconstructed by stretching it linearly ${ }^{5}$. The reconstructed image is then calibrated to show the actual dimensions of the beam data. Fig. $3 \mathrm{~b}$ shows the reconstructed image of the $30 \mathrm{~mm} \times 30$ $\mathrm{mm}$ square located in the center of the screen.

By applying this same technique, the relative beam location and spot gize can now be determined by looking at the reconstructed image. This linear reconstruction is not $100 \%$ accurate due to the nature of a linear perspective image. The calibration of the $Y$ axis is accurate, but the $\mathrm{X}$ axis contains an error of approximately $1 \mathrm{~mm}$. This error is produced because reconstruction of the image was done linearly rather than mapping the image to a projection plane parallel to the screen.

After the reconstruction process, the center of the beam can be estimated by searching for the position with peak intensity. This is only an estimate due to the possibility of an intensity interference caused by the grid pattern on the screen. Once the position is found, $\mathrm{X}$ and $\mathrm{Y}$ profiles are plotted at this location. We assume that the electrons are normally distributed, and thus the beam profile can be represented by a Gaussian fit to the $X$ and $Y$ profiles. Fig. $4 a$ and $b$ are the $X$ and $Y$ profiles with a Gaussian fit.

The presence of the grid pattern on the fluorescent screen aids in the calibration during reconstruction of the image. It also degrades the quality of the image data by imposing a grid pattern on the image. In order to compensate for the error caused by this grid pattern, the discrete patterns of the $\mathrm{X}$ and $\mathrm{Y}$ profiles are eliminated by joining all the peaks to form envelopes. Since the $X$ and $Y$ profiles of the beam image should be continuous curves without the grid patterns, these envelopes better represent the original beam image. Figs. $4 \mathrm{c}$ through $\mathrm{f}$ show the envelope representation of the original beam image.

The center lines on these figures indicate the center of peak intensity of the beam. The dotted lines represent the position of the full width half max (FWHM). The FWHM is the position where the intensity of the beam is half of the peak value. The calculated $\mathrm{X}$ and $\mathrm{Y}$ locations represent the relative position rather the absolute position. They are compared to other beam data to determine the relative changes. 


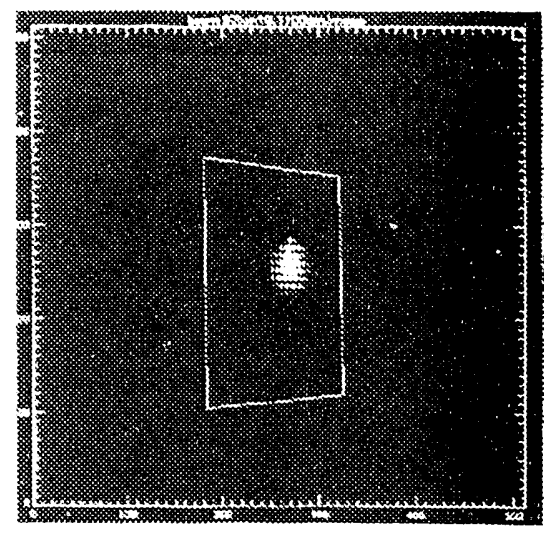

(a.) Beam Image

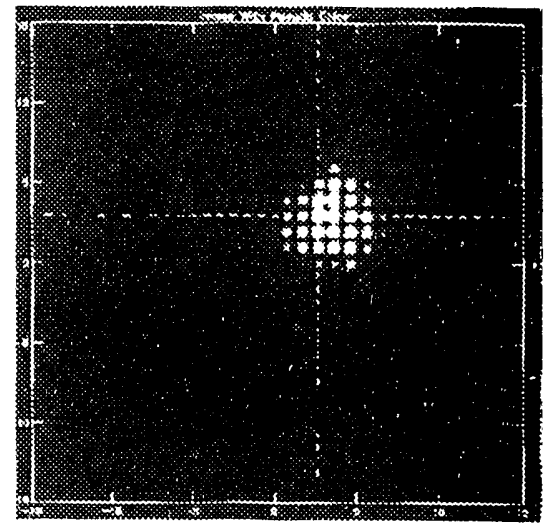

(c.) Reconstructed Image

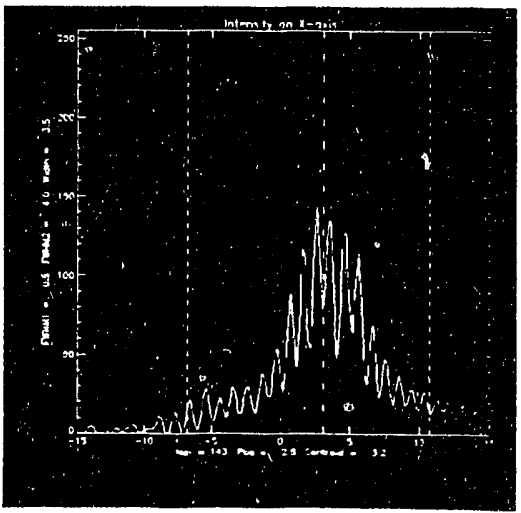

(e.) X Profile at the Estimated Center

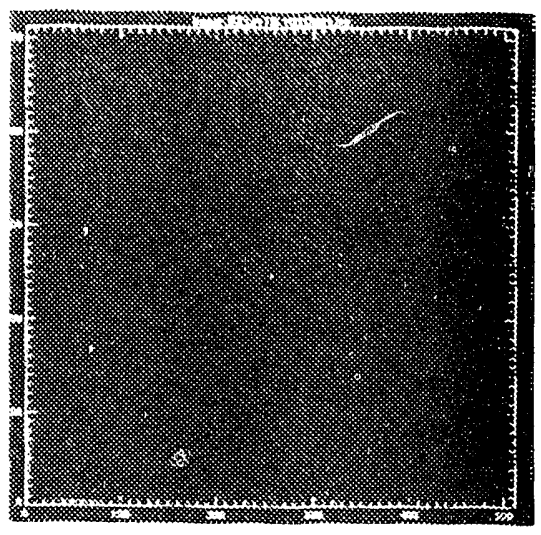

(b.) Background

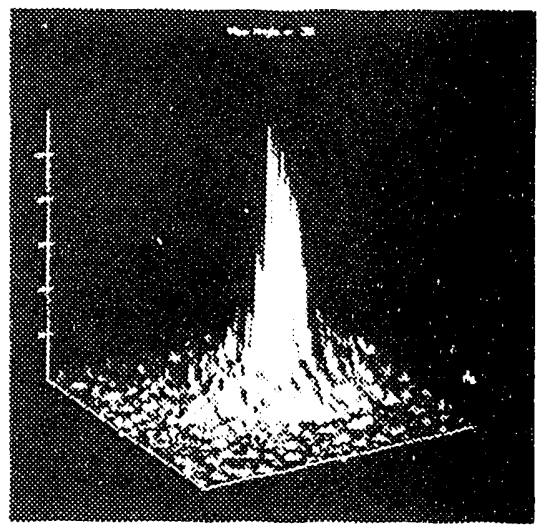

(d.) 3D Projection Reconstructed Image

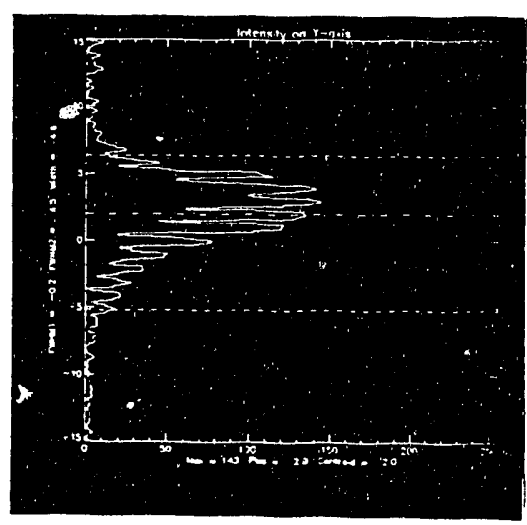

(f.) Y Profile at the Estimated Center

Fig. 1 Typical Output of PV-Wa:e Anal::is 


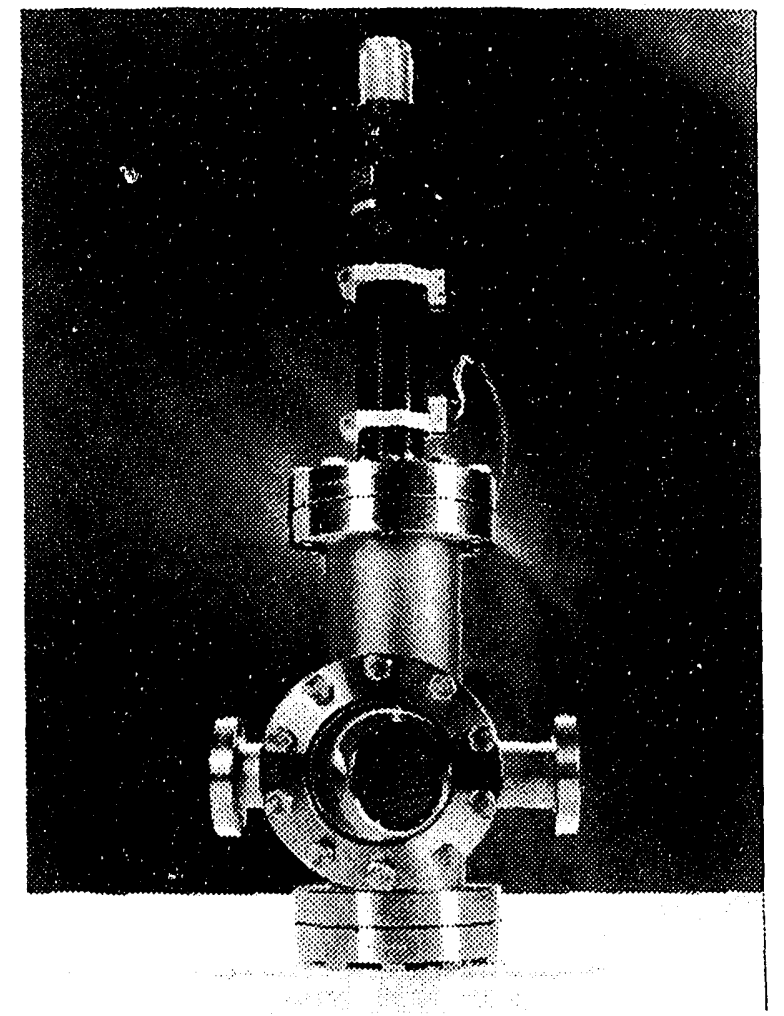

Fig. 2 Fluorescent Screen Assembly

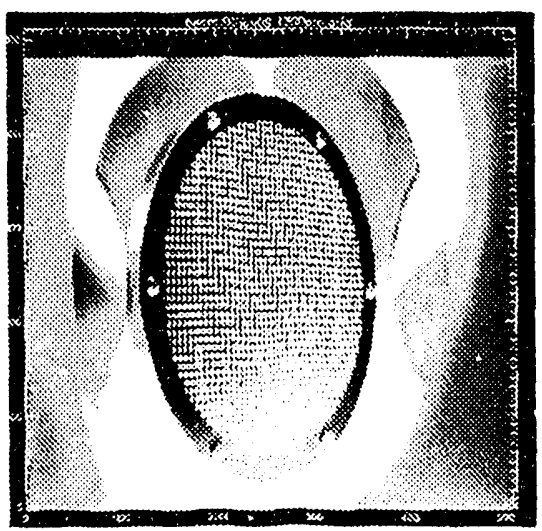

(a.) Original Screen Image

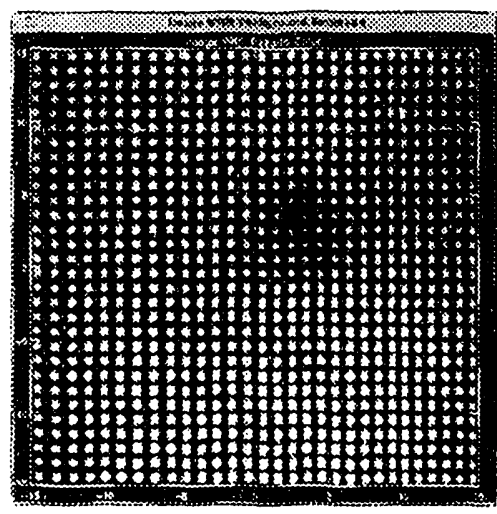

(b.) $30 \mathrm{~mm} \times 30 \mathrm{~mm}$ square located at the center of the screen

Fig. 3 Reconstruction of distorted Screen Image 


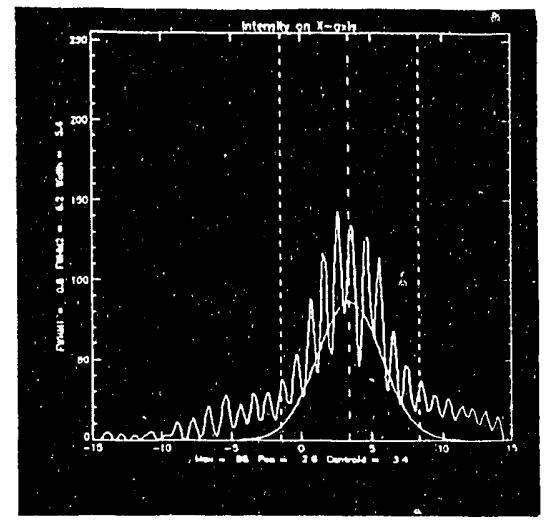

(a.)

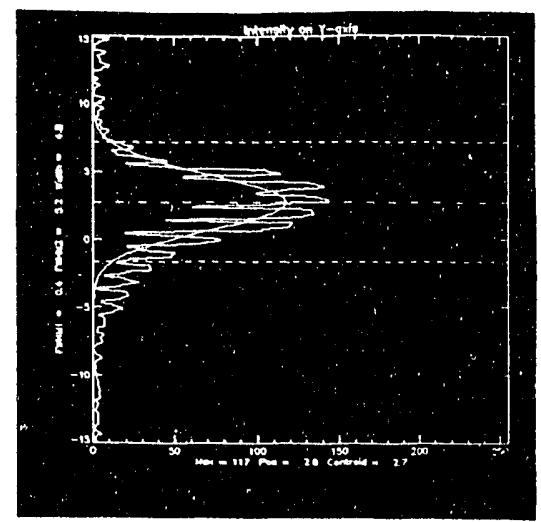

(b.)

Gaussian Fit on $\mathrm{X}$ and $\mathrm{Y}$ Profiles

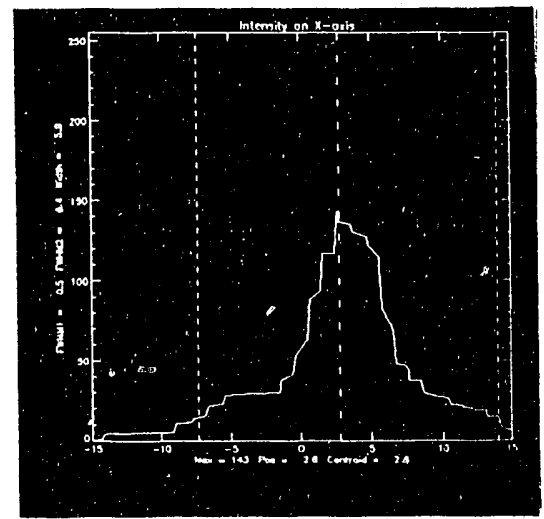

(c.)

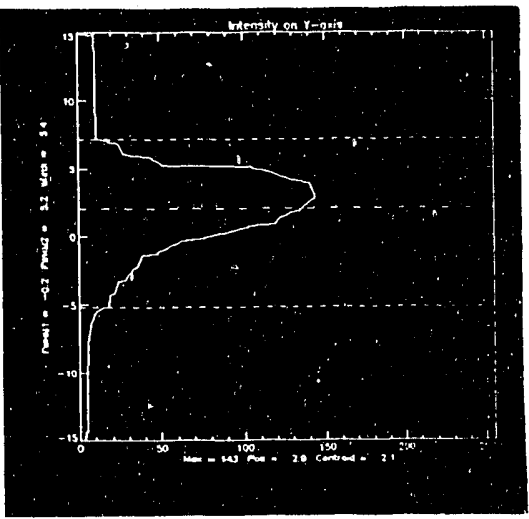

(d.)

Envelope Compensation on $\mathrm{X}$ and $\mathrm{Y}$ Profiles

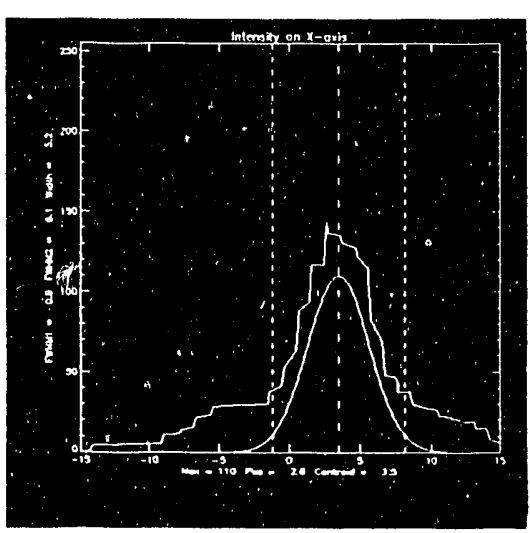

re.

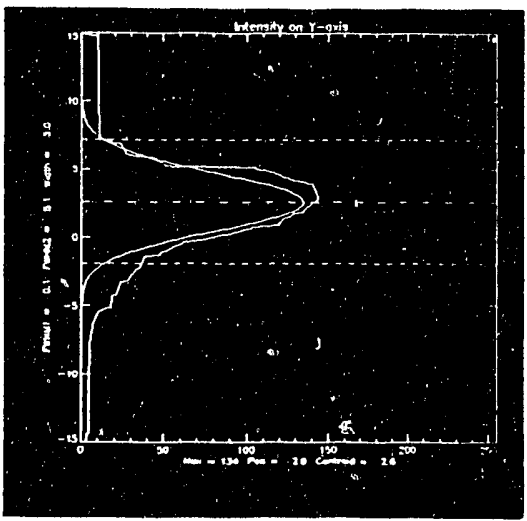

If

Gaussian Fit and Envelope Compensation on $\mathrm{X}$ and $Y$ Profiles

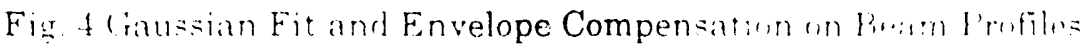




\section{CONCLUSION}

The current frame grabber system of the linac test stand is a preliminary setup to gain experience and identify problems before the final installation of the APS linac. The frame grabber system and image processing procedures will be upgraded for use in the actual APS linac.

The design of the fluorescent screen will be changed. Instead of having a grid pattern on the screen, only cross-hairs will be used to indicate the screen's center. The grid pattern adequately eliminates surface charge buildup, but interferes with the beam's image. We would like to eliminate the grid for that reason and are currently investigating other charge dissipation techniques. The timing of the frame grabber will be improved by direct synchronization to the electron gun trigger. A camera with external synchronization will be used to eliminate the timing uncertainty. Finally, the current image reconstruction procedure will be changed. Linear perspective distortion will be taken into account during reconstruction. The image will be mapped to the projection plane parallel to the screen rather than just shaping it linearly.

1. Precision Visuals Inc., PV-WAVE Command Language Version 3.1 Technical Reference Manual (1991).

2. C.D. Johnson, The Development and Use of Alumina Ceramic Fluorescent Screens, CERN, Geneva, Switzerland (1990).

3. Datacube Inc., DigiMax - Digitizer and Display Device Hardware Reference Manual (1990).

4. Datacube Inc., RoiStore - Region of Interest Storage Device Hardware Reference Manual (1990).

5. Foley, Van Dam, Feiner, and Hughes, Computer Graphics Principles and Practice, 2nd Edition, Addison-Wesley (1990).

*Work supported by the U.S. Department of Energy Office of

Basic Energy Sciences, under Contract No. W-31-109-ENG-38.

\section{DISCLAIMER}

This report was prepared as an account of work sponsored by an agency of the United States Government. Neither the United States Government nor any agency thereof, nor any of their employees, makes any warranty, express or implied, or assumes any legal liability or responsibility for the accuracy, completeness, or usefulness of any informa:ion, apparatus, product, or process disclosed, or represents that its use would not infringe privately owned rights. Referprocess disclosed, or represents that its use would not in specific commercial product, process, or service by trade name, trademark, manufacturer, or otherwise does not necessarily constitute or imply its endorsement, recommendation, or fayoring by the United States Government or any agency thereof. The views and opinions of authors expressed herein do not necessarily state or reflect those of the United States Government or any agency thereof. 

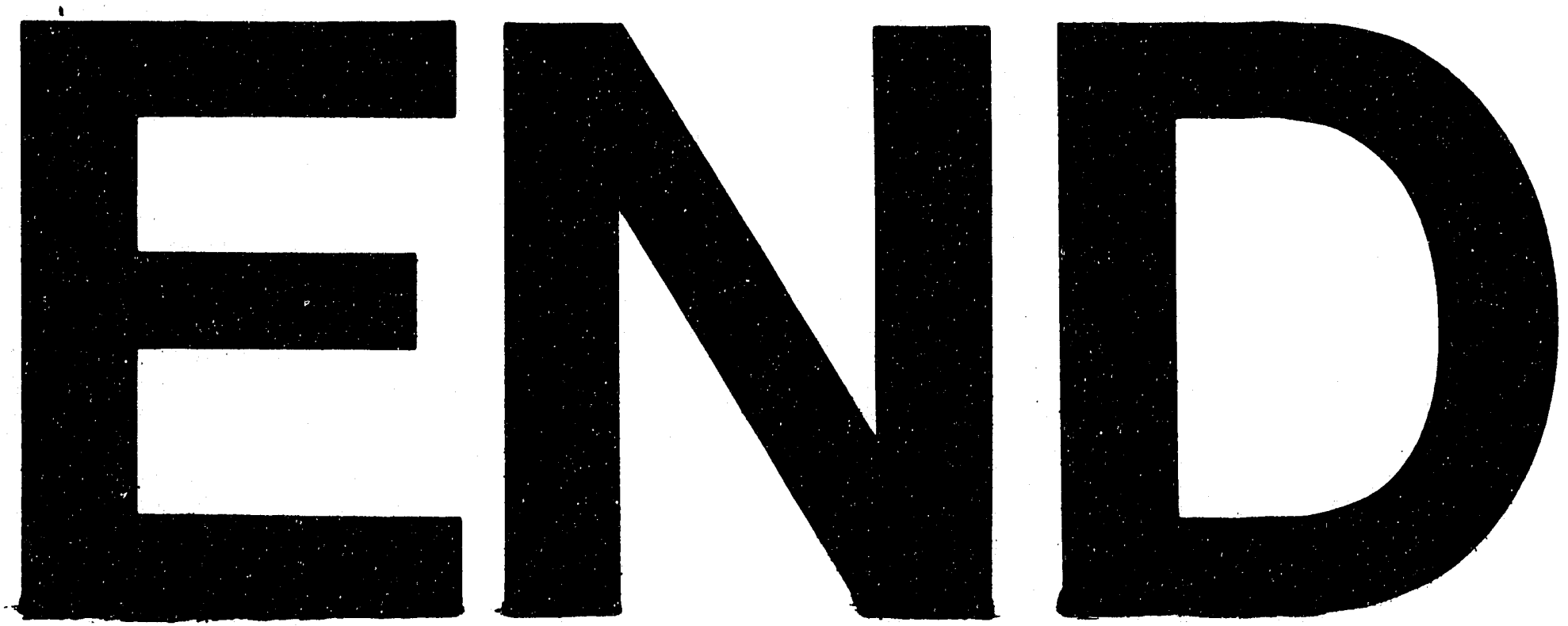

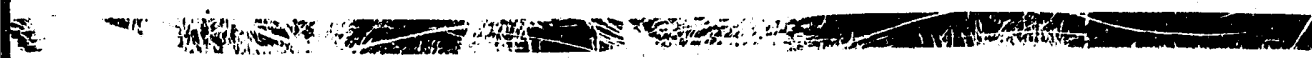
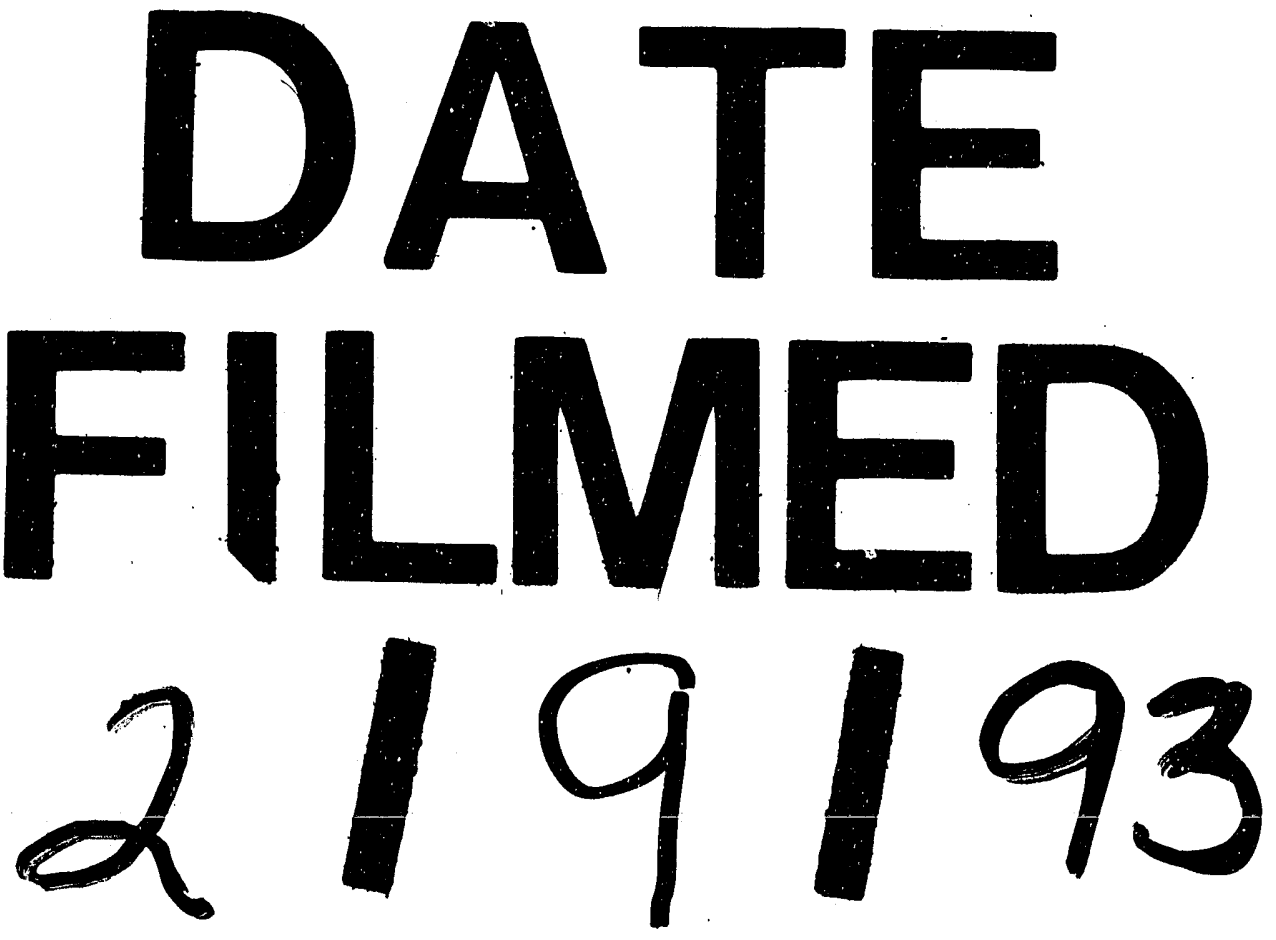
